\title{
A Cyclic Attribution Technique Feature Selection Method for Human Activity Recognition
}

\author{
Win Win Myo, Wiphada Wettayaprasit, and Pattara Aiyarak* \\ Department of Computer Science, Faculty of Science \\ Prince of Songkla University, Hat Yai, 90110, Thailand \\ E-mail: winwinmyo76@gmail.com,wiphada.w@psu.ac.th, pattara.a@psu.ac.th
}

Received: 29 April 2019; Accepted: 07 June 2019; Published: 08 October 2019

\begin{abstract}
Feature selection is a technique of selecting the most important features for predictive model construction. It is a key component in machine learning for many pattern recognition applications. The primary objective of this paper is to create a more precise system for Human Activity Recognition (HAR) by identifying the most appropriate features. We propose a Cyclic Attribution Technique (CAT) feature selection technique for recognition of human activity based on group theory and the fundamental properties of the cyclic group. We tested our model on UCI-HAR dataset focusing on six activities. With the proposed method, 561 features could be reduced to 63. Using an Artificial Neural Network (ANN), we compared performances of our new dataset with selected features and the original dataset classifier. Results showed that the model could provide an excellent overall accuracy of $96.7 \%$. The proposed CAT technique can specify high-quality features to the success of HAR with ANN classifier. Two benefits support this technique by reducing classification overfitting and training time.
\end{abstract}

Index Terms-Feature selection, Attribution technique, Human activity recognition, Cyclic group, Artificial Neural Network.

\section{INTRODUCTION}

Feature or attribute selection is a process of searching the important features in a specified classification problem from the original dataset for model construction, also known as a variable selection. Due to the improved performance of good features, feature selection is an essential part of machine-learning problems. A wellselected feature set not only improves the performance of recognition but also reduces the computational complexity [1]. Feature selection can remove irrelevant or redundant features that cause a false output of classifier without including much special information [2].

Human Activity Recognition (HAR) has received important attention in the field of artificial intelligence over the previous decade as it is essential in various applications such as healthcare systems, sports, and security system [3]-[5]. As the difficulty of the process and overlarge dataset in HAR, the feature selection is an essential part of HAR [6]. Although research groups have been reached to develop a general, stable, and accurate process in the recognition of human activity, the predictive performance is still lacking. Irrelevant or redundant features cause not only the complexity of model in training and testing samples but also the difficulty in getting robust performance [7]. In this article, this is the primary challenge.

Feature selection is a complicated and difficult process in machine learning. It needs well-defined determinations with multi-way dependencies and correlations, which means choosing the best independent features may not create the best feature set. In this sense, we propose a Cyclic Attribution Technique (CAT) to evaluate good features for constructing a better predictive model on HAR based on the group theory and the fundamental properties of the cyclic group. We tested the CAT on UCI-HAR dataset [8] using Artificial Neural Network (ANN) classifier. We demonstrate the effectiveness of this model by comparing performances with original dataset.

The main contributions of this article are to design the CAT as a new feature selection method for more effective and robust classification in machine learning and to improve the performance classification in HAR. Two benefits support this technique by 1) reducing the overfitting and 2) training time on classification. The purpose of this technique is to improve the performance on HAR as well as several machine learning problems. Results showed that the CAT feature selection method discovers the most important features and removes 498 features from 561 with better accuracy of $96.7 \%$ in HAR. According to the results, we suggest that the CAT is a better, more precise and more efficient technique of selecting features for the recognition of human activity.

The remaining of the article is organized as the following. Section 2 discusses other researchers ' associated works and section 3 presents the theory of context. The methodology for this study is introduced in section 4 . Section 5 analyzes the experimental results and discusses them. Finally, section 6 concludes the article.

\section{RELATED WORKS}

Feature selection has become an important role in many machine learning problems. This article concludes 
some important feature selection methods widely used in the past decade. There are three types of feature selection techniques: embedded method, filter method, and wrapper method. Filter methods are applicable for any types of machine learning problems and act faster than wrapper methods. However, wrapper methods for the classifier hypothesis can control feature dependencies and are more effective than the filter method. The choice of features in HAR is more essential as the data with redundant and useless information captured by sensors. To introduce a new feature selection method, W. Win Myo et al. [7] used a linearly dependent concept and improved robustness accuracy in HAR. L.Wang et al. [9] studied the generalized discriminant analysis feature reduction method to develop the determination of the physical activity of human using wearable sensors. Since the feature selection is also an important technique in the healthcare system, Z Zhang et al. [10] introduced a novel disease-specific feature selection method using the dynamic time wrapping for automatic heartbeat classification. Y. Guo et al. [11] suggested a new feature selection method based on tensor, demonstrating the evidence of their proposed method.

To perform feature and instance selection, J. Derrac et al. [12] investigated an evolutionary model for a feature and instance selection using a co-operative coevolutionary algorithm and approved this method in many computational problems. Data Dimensionality Reduction (DDR) is also an essential process in human activity recognition. The data dimension reduction technique was implemented by M. Simão et al. [13] to reduce incomplete data by using re-sampling raw data and principal component analysis. J. Kersten et al. [14] suggested a new expectation-maximization algorithm for feature selection in supervised classification problems by using simultaneous feature selection and Gaussian mixture estimation and showed that better results could be achieved with this strategy. Feature selection can reduce not only original features but also the data dimension such as principal component analysis, or linearly discriminant analysis. In recent years, many feature selections techniques in machine learning problems have been developed, but still difficulties to enhance feature selection methods for determining human activity. U. Maurer et al. [3] used the minimum redundancy maximum relevance feature selection method to design activity recognition. Using Relief-F algorithms, fast correlation-based filter, and correlation-based feature selection, J. Howcroft et al. [15] showed the importance of feature selection for elderly fall risk and faller classification.

In machine learning problems, a classifier has an important role to learn and predict input data. According to the literature reviews, k-nearest neighbor, artificial neural network, and decision tree are well-known classifiers for HAR. In this viewpoint, W. Win Myo et al. [16] suggested and built a new classifier using artificial neural networks HAR. Many studies on the determination of human activity as carried out using machine learning techniques. L. Cao et al. [17] suggested an efficient group-based context-aware classification method for human activity recognition using smartphones They achieved the best accuracy comparing with other popular classifiers. M. Hassan et al. [18] used a kernel principal component analysis and linear discriminant analysis to extract the effective features. They trained a deep belief network and compared the results with traditional artificial neural network and supported vector machine classification methods. They demonstrated greater precision in their technique than other classifiers. Many researchers attempted to get accurate performance from the requirements of classification in determining human activity. D. Acharjee et al. [19] presented by accelerometer and gyroscope in mobile phone a fast and accurate model of the hidden Markov model classifier for the multiple activities of the single user. The thresholdbased condition box was also intended by D. Acharjee et al. [20] to minimize feature vectors using accelerometer and gyroscope. They had focused on the type of sensors to be used in human activity determination.

\section{BACKGROUND THEORIES}

Group theory, a branch of abstract algebra, is an essential tool in several scientific fields including mathematics, physics, chemistry, etc. It is the study of symmetry for anything that stays invariant under some transformations [21]. Group theory can predict the existence group elements. Different object structure and behavior have different symmetries. For the asymmetry of polygons, group theory is very essential in geometry. Group theory is implemented in number theory and elliptic groups are widely applied in modern cryptography fields.

A cyclic group is an abelian group generated by a single element. In group theory, the fundamental properties of cyclic groups play a vital role. M. Eie et al. [22] considered the fundamental of a cyclic group and classified the cyclic group. The authors discussed about the importance of stars polygon in art and culture [23]. The study of abstract groups challenges the transformation to algebraic ideas. By using star polygons, S. Spitzer [24] tried to understand the cyclic group structure for algebra ideas. M. M. El-Farrah et al. [25] proposed a technique for finding expected numbers of cyclic groups. The relationship between cyclic soft groups and classical groups was researched by H. Aktaş et al. [26]. Von Zur Gathen et al. suggest a method for finding an average order of the elements in a cyclic group of order $n$. G. Oman et al. [27] demonstrated that an arbitrary group $\mathrm{G}$ is cyclic if and only if there are distinct subgroups with distinct indexes in $\mathrm{G}$.

Group theory and its properties are widely used in many areas such as physics, chemistry, biochemistry, medicine, engineering and mathematics themselves. There are many complex classes with different kind of elements in a group. A classification of the membership of that group is one of the most important points in a group. Sometimes, groups contain some arbitrary subgroups without particular properties. At times, the 
groups could also come with some binary operations with special properties.

For this work, we are interested in group theory and fundamental properties of the cyclic group with a binary operation involving some special properties, which are shown as the following definitions.

\section{Definition (1)}

Let $\mathrm{G}=\left\{\mathrm{g}_{1}, \mathrm{~g}_{2}, \ldots, \mathrm{g}_{\mathrm{n}}\right\}$ be a finite set of elements. Then $\mathrm{G}$ is called a group if it is satisfying four properties: closure, associativity, unity, and inverse under addition operation.

\section{Definition (2)}

Let $\mathrm{G}$ be a group. Then order of a group $\mathrm{G}$ is the total number of elements in $\mathrm{G}$ denoted by $|\mathrm{G}|$ or $\mathrm{O}(\mathrm{G})$.

For example:

Given $\mathrm{G}=\{1,2,3,4,5,6,7\}$,

then order of a group $G$ is $|G|=O(G)=7$.

\section{Definition (3)}

Let $\mathrm{G}$ be a group. Then $\mathrm{G}$ is a cyclic group

if $\mathrm{G}=\langle a\rangle=\{n a / a \in \mathrm{G}, n \in Z\}$, which is called a cyclic group generated by $a$.

For example:

Given $\mathrm{G}=\{1,2,3,4,5,6,7$,$\} and \mathrm{Z}_{5}=\{2,3,4,5,6\}$, then $Z_{5}=\langle 1\rangle$ with $|1|=5$.

Here, we call $Z_{5}=\langle 1>$ a cyclic group generated by 1 .

\section{Definition (4)}

Every cyclic group satisfies the following fundamental properties:

Every group is itself a cyclic group with generator 1 .

Every subgroup of a cyclic group is also a cyclic group.

The order of every subgroup is a divisor of group $G$ order.

For each divisor $k$ of the order of $G$, there exists exactly one subgroup of order $\mathrm{k}$.

For example:

Given $\mathrm{G}=\mathrm{Z}_{8}=\{1,2,3,4,5,6,7,8\}$,

then $\mathrm{G}$ is cyclic group generated by 1 with $|\mathrm{G}|=8$

and divisors of 8 are 2 and 4 .

Since, each divisor has exactly one subgroup of $\mathrm{G}$.

$\langle 2\rangle=\{2,4,6,8\}$ with order 4 and

$\langle 4\rangle=\{4,8\}$ with order 2 are subgroups of $G$.

Definition (5)

The function of Euler Phi $\varphi$ is described in a cyclic group as the total number of elements. If there is a divisor $\mathrm{d}$ of the order of group $\mathrm{G}$, then the number of elements of that devisor $\mathrm{d}$ can be expressed as. $\varphi=$ the number of positive integers less than $\mathrm{d}$ and relatively prime to $d$

Example is shown in Table 1.

Table 1. Evaluation for the total number of elements of cyclic subgroup $\mathrm{G}$

\begin{tabular}{|c|c|c|}
\hline$|\mathrm{G}|$ & $d$ & $\varphi$ \\
\hline$($ Order of $\mathrm{G})$ & (Divisor of $|\mathrm{G}|)$ & (Euler Phi) \\
\hline 4 & 2,4 & 1,2 \\
\hline 6 & 2,3 & 1,2 \\
\hline 8 & $2,4,8$ & $1,2,4$ \\
\hline 10 & 2,5 & 1,4 \\
\hline
\end{tabular}

\section{METHODOLOGY}

There are five main processes in this study: 1) using UCI-HAR dataset, 2) pre-processing data, 3) implementing the proposed technique of CAT feature selection, 4) classification with $\mathrm{ANN}$, and 5) performance evaluation. Fig 1 demonstrates the system design using Cyclic Attribution Technique (CAT) feature selection method to recognize human activity.

\section{A. UCI-HAR dataset}

The study carried out UCI-HAR dataset that the researchers of the University of California of Irvine produced in 2013 [8]. It is the most popular dataset for UCI repository (available from http:/archive.ics.uci.edu/ml). In this dataset, accelerometer sensor and gyroscope sensor of the Samsung Galaxy SII mobile phone were utilized to collect the data. With a constant rate of $50 \mathrm{~Hz}$, the raw data was sampled, further analyzed and extracted features.

Thirty volunteers performed six activities (walking, walking upstairs, walking downstairs, sitting, standing, and laying) within an age bracket of 19-48 years. Raw data appeared as a series of 10299 instants containing a timestamp, three accelerometer values along the $\mathrm{X}$-axis, $\mathrm{Y}$-axis, $\mathrm{Z}$-axis and three gyroscope values along the $\mathrm{X}$ axis, Y-axis, Z-axis. Two domains obtained the complete 561 features from time domain and frequency domain. The detailed description of features in the UCI-HAR dataset is shown in Table 3.

\section{B. Data Preprocessing}

The UCI-HAR dataset is divided into three data subgroups of features by the appropriate domain (time domain and frequency domain) as shown in Fig 2. In which time domain as behaviors of extracted time features was split into two feature groups.

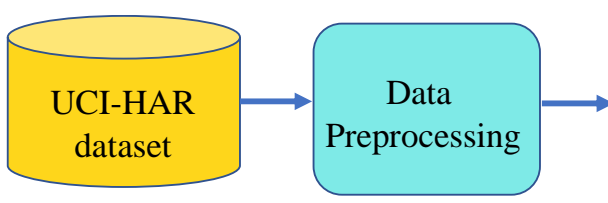

(A)
(B)

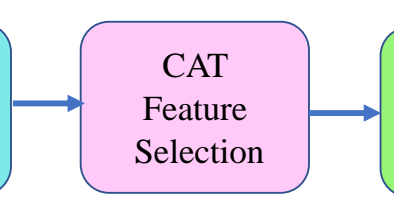

(C)

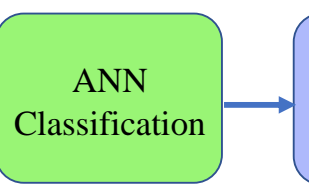

(D)
Performance evaluation

Fig.1. System design using the proposed Cyclic Attribution Technique feature selection method 
Fig 2 contains three subgroups of data: 1) $\mathrm{T}$ is data with time domain-relevant features based on variable values; 2) $\mathrm{A}$ is data with time domain features based on angle values; 3) $\mathrm{F}$ is data with frequency domain-relevant features.

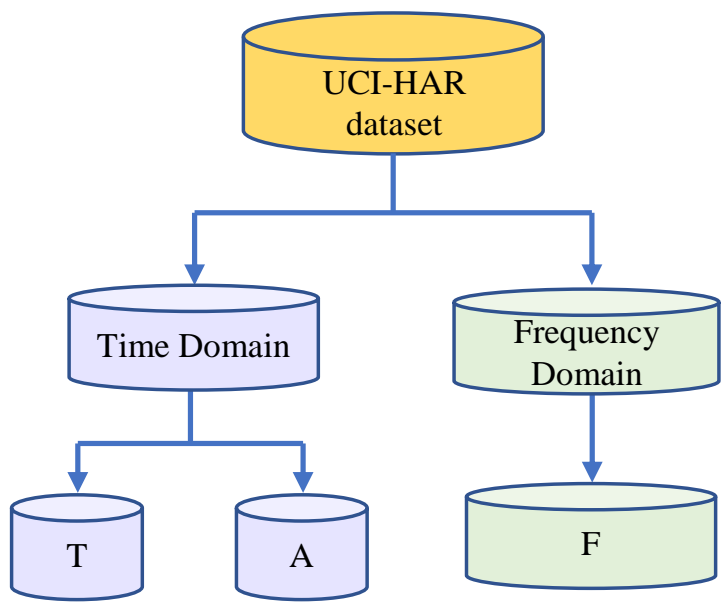

Fig.2. Splitting to three data subgroups of features

The total number of features with relevant $\mathrm{T}, \mathrm{A}$, and $\mathrm{F}$ is demonstrated in Table 2. according to the separate data subgroups.

Table 2. Separating three feature categories from UCI-HAR dataset

\begin{tabular}{cccc}
\hline Notation & $\begin{array}{c}\text { Feature } \\
\text { Domain }\end{array}$ & $\begin{array}{c}\text { No. } \\
\text { Instances }\end{array}$ & $\begin{array}{c}\text { No. } \\
\text { Features }\end{array}$ \\
\hline T & Time & 10299 & 265 \\
A & Time & 10299 & 7 \\
F & Frequency & 10299 & 289 \\
\hline
\end{tabular}

Table 3. Detail categorization of feature groups from UCI-HAR dataset

\begin{tabular}{|c|c|c|}
\hline Category & Signals Types & $\begin{array}{c}\text { No. } \\
\text { Features }\end{array}$ \\
\hline \multirow{10}{*}{$\begin{array}{l}\text { Time Domain } \\
\text { Feature }(265)\end{array}$} & tBodyAcc-XYZ & 40 \\
\hline & tGravityAcc-XYZ & 40 \\
\hline & tBodyAccJerk-XYZ & 40 \\
\hline & tBodyGyro-XYZ & 40 \\
\hline & tBodyGyroJerk-XYZ & 40 \\
\hline & tBodyAccMag & 13 \\
\hline & tGravityAccMag & 13 \\
\hline & tBodyAccJerkMag & 13 \\
\hline & tBodyGyroMag & 13 \\
\hline & tBodyGyroJerkMag & 13 \\
\hline $\begin{array}{l}\text { Time Domain } \\
\text { Feature (7) }\end{array}$ & angle() & 7 \\
\hline \multirow{7}{*}{$\begin{array}{c}\text { Frequency } \\
\text { Domain Feature } \\
(289)\end{array}$} & fBodyAcc-XYZ & 79 \\
\hline & fBodyAccJerk-XYZ & 79 \\
\hline & fBodyGyro-XYZ & 79 \\
\hline & fBodyAccMag & 13 \\
\hline & fBodyAccJerkMag & 13 \\
\hline & fBodyGyroMag & 13 \\
\hline & fBodyGyroJerkMag & 13 \\
\hline \multicolumn{2}{|c|}{ Total features } & 561 \\
\hline
\end{tabular}

\section{Cyclic Attribution Technique (CAT))}

The main aim of this research is to design a novel feature selection classifier, named Cyclic Attribution Technique (CAT), for development of the prediction performance in HAR. The proposed feature selection method will look carefully at the most important features from original features. Before developing a CAT, three new definitions using group theory and the cyclic group characteristics are introduced, which are interesting concepts and methods used in this feature selection method.

\section{Definition (6)}

Let a group $\mathrm{G}$ be a set containing $\mathrm{N}$ features and it is a cyclic group itself with generator 1 . Let $D$ be a divisor of $\mathrm{N}$ and $Q$ be a quotient of $\mathrm{N}$. Then a cyclic group $\langle Q\rangle$ is a new feature set containing relevant or useful features of the dataset.

\section{Definition (7)}

Let a cyclic group $\langle Q\rangle$ be a set of selected features containing relevant or useful features of the dataset. Then, the number of features of this new feature set ' $\langle Q\rangle$ ' is

$$
\varphi(Q)=Q\left(1-\frac{1}{q_{1}}\right)\left(1-\frac{1}{q_{2}}\right) \ldots\left(1-\frac{1}{q_{h}}\right)
$$

Where $q_{1}, q_{2}, \ldots, q_{h}$ are prime divisors of $Q$ and $\mathrm{h}<Q$.

Definition (8)

Let $D$ be a divisor of $\mathrm{N}, \varphi(Q)=m$ and $\langle Q\rangle$ be a set of selected features. The selected features are

$$
<Q>=\{D * i / i=1,2, \ldots, m\}
$$

The creating of the Cyclic Attribution Technique model consists of four primary procedures. The first process is to find mean values of all features and sort them in ascending order. The second process is to determine new feature subgroups and their number of features. The third process is to search all features of the new group of features. The fourth process is to group the new features.

\section{Finding and sorting mean values of all features}

The mean value of each feature is calculated by the following equation according to three different feature groups (T, A, F) from Table 2 .

$$
\text { Mean }=\frac{\sum_{i=1}^{n} x_{i}}{n}
$$

where $x_{i}$ is a time series value of each feature and $\mathrm{n}$ is the total number of each feature group.

According to equation (1), all features from three different groups ( $\mathrm{T}, \mathrm{A}$, and $\mathrm{F}$ ) are listed by hierarchic relation of mean value as $f_{i}<f_{j}$ where fi and $\mathrm{fj}$ are features. 
For all hierarchical features, the following transitivity holds:

$$
\left(f_{i}<f_{j}\right) \Lambda\left(f_{i}<f_{h}\right) \rightarrow f_{i}<f_{h}
$$

Then, we renamed all features in ascending order as follows:

$$
\begin{aligned}
& \mathrm{T}=\left\{\mathrm{t}_{1}, \mathrm{t}_{2}, \ldots, \mathrm{t}_{265}\right\}, \\
& \mathrm{F}=\left\{\mathrm{f}_{1}, \mathrm{f}_{2}, \ldots, \mathrm{f}_{289}\right\}, \text { and } \\
& \mathrm{A}=\left\{a_{1}, a_{2}, \ldots, a_{7}\right\} .
\end{aligned}
$$

where $\mathrm{T}$ contains 265 features from the time domain, $\mathrm{F}$ contains 289 features from the frequency domain, and A contains 7 features from time domain.

\section{Determining new feature sets and number of elements offeature sets}

To complete this process, Algorithm (1) performs on three different features subgroup from $\mathrm{T}$ (time domain), $\mathrm{F}$ (frequency domain), and A (Time domain) as shown in Fig 3.

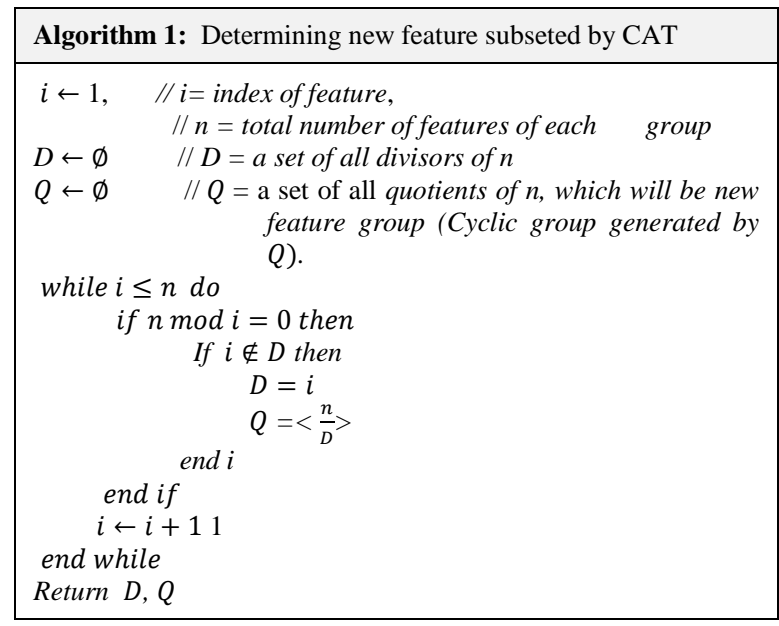

Fig.3. Determining new features sets
The proposed definition 6 generalizes Algorithm 1 as shown in Fig 3. Input ' $n$ ' to this algorithm is the total number of features of three different feature groups. Output ' $D$ ' is the set of the divisors of input ' $n$ '. Output ' $Q$ ' is the set of the quotients of input ' $n$ ', which is a cyclic group with generator ' $Q$ '. On the other hand, ' $\langle Q\rangle$ ' is the desired new features set of our study.

\section{Searching for new feature sets}

The following Algorithm (2) is generalized by the proposed definitions (7-8) as described in Fig 4. The value of $\varphi$ is the total number of features of the new feature subset produced by definition (7).

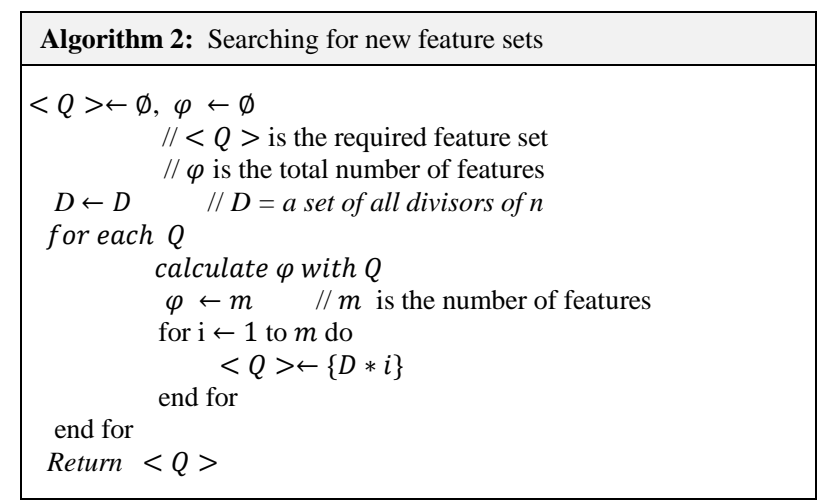

Fig.4. Searching for new feature sets

In Algorithm 2, Input ' $D$ ' and ' $Q$ ' are from Algorithm 1. Output ' $\langle Q\rangle$ ' is the desired new feature set of our study.

Now, our suggest CAT method effectively chooses four critical feature sets: T1, T2, F1, and A1 from UCIHAR dataset as shown in Table 4. In which, feature sets from time domain are selected for $\mathrm{T} 1$ and $\mathrm{T} 2$. F1 is a

\begin{tabular}{|c|c|c|c|c|}
\hline $\begin{array}{l}\mathrm{G} \text { (Feature } \\
\text { group) }\end{array}$ & $\begin{array}{c}D=\text { All divisors } \\
\text { of } \mathrm{T}, \mathrm{F}, \mathrm{A}\end{array}$ & $\begin{array}{c}\langle Q\rangle=\text { subgroup } \\
\text { of } \mathrm{G}=\text { new } \\
\text { features set }\end{array}$ & $\begin{array}{c}\varphi=\text { number of } \\
\text { elements of } \\
\text { new features set }\end{array}$ & $\begin{array}{l}\text { Desired elements } \\
\text { for new features set }\end{array}$ \\
\hline \multirow{2}{*}{ (265) } & 53 & $\mathrm{~T} 1=\langle 5\rangle$ & 4 & $\{53,106,159,212\}$ \\
\hline & 5 & $\mathrm{~T} 2=\langle 53\rangle$ & 52 & $\{5,10,15,20,25,30,35,40,45,50,---, 250,255,260\}$ \\
\hline $\mathrm{F}(289)$ & 17 & $\mathrm{~F} 1=\langle 17\rangle$ & 17 & $\begin{array}{c}\{17,34,51,68,85,102,119,136,153,170,187,204,221,238, \\
255,272,289\}\end{array}$ \\
\hline$A(7)$ & 7 & $\mathrm{~A} 1=\langle 7\rangle$ & 7 & $\{1,2,3,4,5,6,7\}$ \\
\hline
\end{tabular}
selected feature from frequency domain and $\mathrm{A} 1$ is selected features from remaining time domain.

Table 4. Evaluating newly feature subsets and their features by the CAT

In Table $4, \mathrm{D}$ is the divisors set of input $\mathrm{n}$ where $\mathrm{n}=$ total number of features, $Q$ is the quotients set of input $n$, and $\varphi$ is the total number of features for new subset of features where T1, T2, F1, and A1 are new feature subsets.

\section{Grouping the CAT features}

The newly selected features (T1, T2, F1, A1) by the proposed CAT method are grouped by combination rule to evaluate the effectiveness of the CAT model using classifier as illustrated in Table 5. The combination with the newly selected feature sets includes fifteen groups from $\mathrm{G} 1$ to $\mathrm{G} 15$.

\section{ANN Classification}

To determine the effectiveness of CAT model, the ANN model is designed with a feed-forward propagation algorithm. ANN used two hidden layers with a learning 
rate of 0.0005 . All selected available data is divided into $70 \%$ for training set and $30 \%$ for testing. Finally, the ANN model based on CAT is prepared to classify the G1 to G15 input feature sets.

\section{E. Performance Evaluation}

The main aim of this study is to develop a HAD system based the proposed CAT feature selection method. In order to determine the reliability and quality of the CAT method, the performances of six activities (walking, walking upstairs, walking downstairs, sitting, standing, and laying) are evaluated using the ANN classifier.

\begin{tabular}{|c|c|c|c|}
\hline $\begin{array}{l}\text { Groups' } \\
\text { names }\end{array}$ & $\begin{array}{l}\text { Combining all new } \\
\text { feature sets }\end{array}$ & $\begin{array}{l}\text { No. of } \\
\text { features }\end{array}$ & $\begin{array}{c}\text { Average } \\
\text { Accuracy \% }\end{array}$ \\
\hline G1 & $\mathrm{T} 1$ & 4 & 61.42 \\
\hline G2 & $\mathrm{T} 2$ & 52 & 94.63 \\
\hline G3 & $\mathrm{F} 1$ & 17 & 63.01 \\
\hline G4 & A1 & 7 & 72.30 \\
\hline G5 & $\mathrm{T} 1+\mathrm{T} 2$ & 56 & 94.82 \\
\hline G6 & $\mathrm{T} 1+\mathrm{F} 1$ & 21 & 71.88 \\
\hline G7 & $\mathrm{T} 1+\mathrm{A} 1$ & 11 & 85.66 \\
\hline G8 & $\mathrm{T} 2+\mathrm{F} 1$ & 69 & 93.98 \\
\hline G9 & $\mathrm{T} 2+\mathrm{A} 1$ & 59 & 96.34 \\
\hline G10 & $\mathrm{F} 1+\mathrm{A} 1$ & 24 & 91.04 \\
\hline G11 & $\mathrm{T} 1+\mathrm{T} 2+\mathrm{F} 1$ & 73 & 94.43 \\
\hline G12 & $\mathrm{T} 1+\mathrm{T} 2+\mathrm{A} 1$ & 63 & 96.7 \\
\hline G13 & $\mathrm{T} 1+\mathrm{F} 1+\mathrm{A} 1$ & 28 & 92.46 \\
\hline G14 & $\mathrm{T} 2+\mathrm{F} 1+\mathrm{A} 1$ & 76 & 96.31 \\
\hline G15 & $\mathrm{T} 1+\mathrm{T} 2+\mathrm{F} 1+\mathrm{A} 1$ & 80 & 96.34 \\
\hline
\end{tabular}

\section{RESUlTS AND DisCUSSIONS}

\section{A. Experiment 1}

The proposed CAT method successfully selects four critical feature sets from UCI-HAR dataset as T1, T2, F1, and A1. The feature sets, T1, T2, and A1, are new selected feature group from the time domain. F1 is the selected feature set from the frequency domain. The performance of those feature subsets using ANN from each individual domain is described in Table 5.
According to the results in Table 5, T2 obtained the best accuracy of $94.63 \%$, which means the features in $\mathrm{T} 2$ are the most sufficient features in HAR. However, T1 is with a lower accuracy of $61.42 \%$, the different performance of T1 and F1 showed only $1.59 \%$ accuracy. Besides, the difference between $\mathrm{A} 1$ and $\mathrm{T} 1$ is $11.66 \%$ accuracy and the difference between $\mathrm{A} 1$ and F1 is $9.07 \%$ accuracy.

\section{B. Experiment 2}

Using the combination rule, all CAT features (T1, T2, $F 1$, and A1) are grouped into from G1 to G15 and the performances are evaluated using ANN to obtain the goal of this study. Table 5 provided the average performance outcomes for each group.

According to the results analyzed in Table 5, G12 combined with A1, T1, and T2 using 63 features achieved the best $96.7 \%$ accuracy. It is found that the frequency domain features make more complex than other types of features. To conclude all results, it is discovered that G12 $(\mathrm{T} 1+\mathrm{T} 2+\mathrm{A} 1)$ obtained the highest accuracy without frequency features. Therefore, using 63 features from G12 gives the best performance for the classification to prove the effectiveness of the proposed model comparing to the performance of the original dataset.

\section{Experiment 3}

In this experiment, the average performances of CAT features are compared with original UCI-HAR dataset features for each domain features using ANN classifier as shown in Table 6.

Table 6. Comparison of average performances between original dataset and the CAT features dataset

\begin{tabular}{|c|c|c|c|c|}
\hline $\begin{array}{c}\text { Feature } \\
\text { Type }\end{array}$ & $\begin{array}{c}\text { UCI-HAR dataset } \\
\text { Features }\end{array}$ & $\begin{array}{c}\text { Accuracy } \\
\%\end{array}$ & $\begin{array}{c}\text { No. } \\
\text { Features }\end{array}$ & $\begin{array}{c}\text { Accuracy } \\
\%\end{array}$ \\
\hline $\begin{array}{c}\text { Time } \\
\text { Domain }\end{array}$ & 265 & 96.12 & 56 & 94.82 \\
\hline $\begin{array}{c}\text { Frequency } \\
\text { Domain }\end{array}$ & 289 & 88.25 & 17 & 63.01 \\
\hline $\begin{array}{c}\text { Another } \\
\text { Domain }\end{array}$ & 7 & 72.08 & 7 & 72.08 \\
\hline $\begin{array}{c}\text { Total } \\
\text { Features }\end{array}$ & 561 & 94.85 & 63 & 96.7 \\
\hline
\end{tabular}

As a result, in Table 6, the accuracy of the CAT features dataset achieved $96.70 \%$ accuracy and UCI-HAR dataset obtained $94.85 \%$ accuracy. This shows that the accuracy of the CAT features dataset is better than the accuracy of the original dataset. In this piece of contribution, the proposed methodology successfully determines the most important 63 features from originally 561 features. 


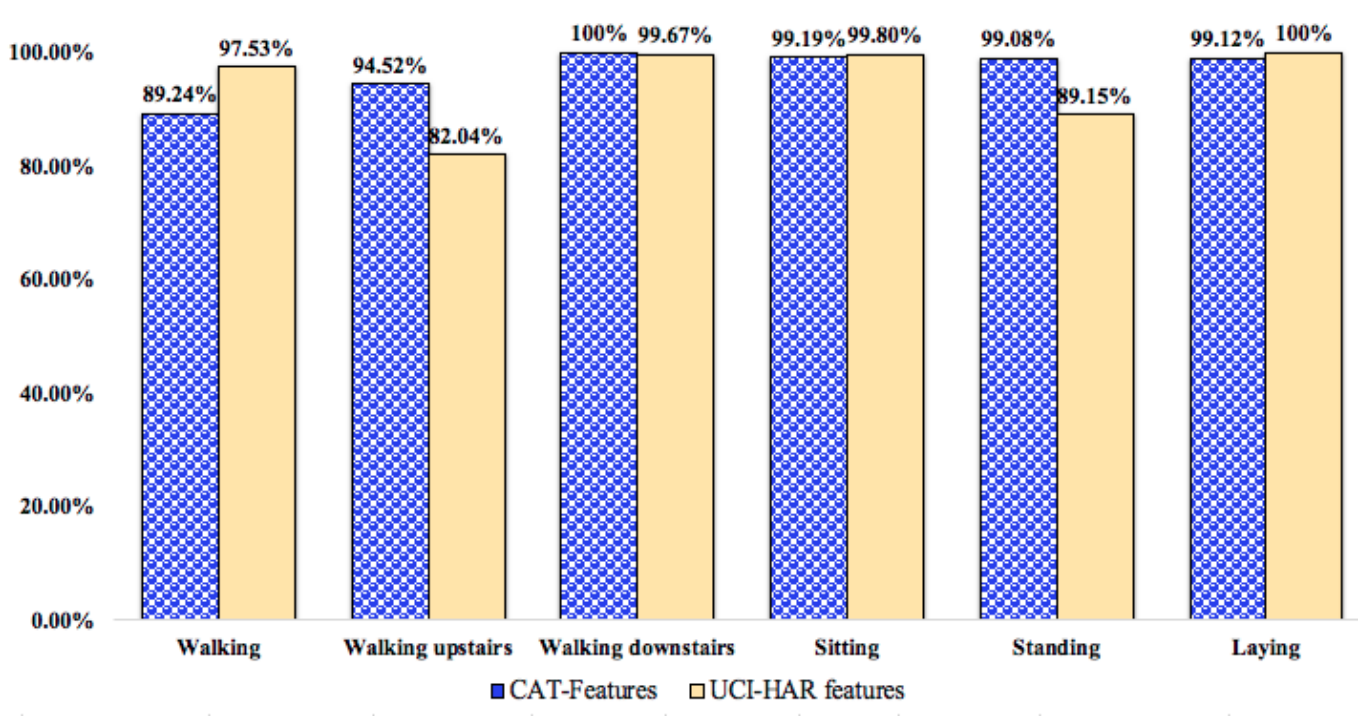

Fig.5. Comparison of performances of six activities with 63 CAT feature set and original dataset

Finally, we compared the performances of six activities (walking, walking upstairs, walking downstairs, sitting, standing, and laying) of the original dataset and the CAT features dataset using the ANN classifier as shown in Fig 5 .

As the result of comparison, the performance of the CAT model is $8.29 \%$ less accurate in walking activity, $0.6 \%$ less accurate in sitting activity, and $0.88 \%$ less accurate in laying activity than the performances of the original dataset. The performance of the CAT model showed higher accuracy in walking upstairs activity with $12.48 \%, 0.33 \%$ higher accuracy in walking downstairs activity, and $9.93 \%$ higher accuracy in sitting activity than the accuracies of UCI-HAR dataset respectively. However, the overall accuracy of the proposed model achieved higher accuracy than the original dataset in classification.

\section{CONCLUSIONS}

The proposed Cyclic Attribution Technique (CAT) feature selection model is based on the special properties of the group theory and the cyclic group. The model correctly selected the most important 63 features in UCIHAR dataset and reduced 498 features from 561 features. The CAT highlighted the complexity of frequency features in human activity recognition and the importance of some features from the time domain and frequency domain. It removed all frequency features from the original dataset to endorse the complexity of frequency features. The proposed methodology succeeds in $96.7 \%$ of average classification accuracy. The accuracy of the classification process using ANN claimed the efficiency, reliability, and accuracy of the CAT model. It suggested that the CAT model could be developed in other pattern recognition problems in machine learning.

\section{ACKNOWLEDGEMENT}

This work was supported by the research grant from Graduate School, Prince of Songkla University, Thailand.
It was very much appreciated. The first author would also like to express her appreciation to the PSU President's Scholarship for her Ph.D.

\section{REFERENCES}

[1] G. Forman, "An Extensive Empirical Study of Feature Selection Metrics for Text Classification," Journal of machine learning research, vol. 3, pp. 1289-1305, 2003.

[2] I. Guyon, "An Introduction to Variable and Feature Selection 1 Introduction," Journal of machine learning research., vol. 3, pp. 1157-1182, 2003.

[3] U. Maurer, A. Smailagic, D. P. Siewiorek, and M. Deisher, "Activity recognition and monitoring using multiple sensors on different body positions," Proceedings-BSN 2006: International Workshop on Wearable and Implantable Body Sensor Networks, vol. 2006, no. May 2006, pp. 113-116, 2006.

[4] M. Arif, M. Bilal, and A. Kattan, "Better Physical Activity Classification using Smartphone Acceleration Sensor," Journal of Medical Systems no. September, pp. 0-10, 2014.

[5] I. Suarez, A. Jahn, C. Anderson, and K. David, "Improved Activity Recognition by Using Enriched Acceleration Data," Proceedings of the 2015 ACM International Joint Conference on Pervasive and Ubiquitous Computing (UbiComp'15)., pp. 1011-1015, 2015.

[6] L. Zhen and L. Qiong, "A New Feature Selection Method for Internet Traffic Classification Using ML," Physics Procedia International Conference on Medical Physics and Biomedical Engineering., vol. 33, no. Ml, pp. 13381345, 2012.

[7] W. WinMyo, P. Aiyarak, and W. Wettayaprasit, "A Noble Feature Selection Method for Human Activity Recognition using Linearly Dependent Concept ( LDC )," in the 7th International Conference on Software and Computer Applications, 2018, no. Ldc, pp. 173-177.

[8] D. Anguita, A. Ghio, L. Oneto, X. Parra, and J. L. ReyesOrtiz, "A Public Domain Dataset for Human Activity Recognition Using Smartphones," European Symposium on Artificial Neural Networks, Computational Intelligence and Machine Learning., no. April, pp. 24-26, 2013.

[9] L. Wang, "Recognition of Human Activities with Wearable Sensors," EURASIP Journal on Advances in Signal Processing., vol. 2012, no. 108, pp. 1-13, 2012. 
[10] Z. Zhang, J. Dong, X. Luo, K. S. Choi, and X. Wu, "Heartbeat classification using disease-specific feature selection," Computers in Biology and Medicine.., vol. 46, no. 1, pp. 79-89, 2014.

[11] Y. Guo et al., "Tensor Manifold Discriminant Projections for Acceleration-Based Human Activity Recognition," IEEE Transactions on Multimedia., vol. 18, no. 10, pp. 1977-1987, 2016.

[12] J. Derrac, S. Garc??a, and F. Herrera, "IFS-CoCo: Instance and feature selection based on cooperative coevolution with nearest neighbor rule," Pattern Recognittion., vol. 43, no. 6, pp. 2082-2105, 2010.

[13] M. Simão, P. Neto, and O. Gibaru, "Using data dimensionality reduction for recognition of incomplete dynamic gestures," Pattern Recognition Letters., vol. 0, pp. 1-7, 2017.

[14] J. Kersten, "Simultaneous feature selection and Gaussian mixture model estimation for supervised classification problems," Pattern Recognition., vol. 47, no. 8, pp. 25822595, 2014.

[15] J. Howcroft, J. Kofman, and E. D. Lemaire, "Feature selection for elderly faller classification based on wearable sensors," Journal of Neuro-Engineering and Rehabilitation., vol. 14, no. 1, pp. 1-11, 2017.

[16] W. W. Myo, W. Wettayaprasit, and P. Aiyarak, "Designing Classifier for Human Activity Recognition Using Artificial Neural Network," 2019 4th International Conferrence on IEEE Computer. Communijcation. System, pp. 81-85, 2019.

[17] L. Cao, Y. Wang, B. Zhang, Q. Jin, and A. V. Vasilakos, "GCHAR: An efficient Group-based Context-aware human activity recognition on smartphone," Journal of Parallel and Distributed Computing., 2016.

[18] M. M. Hassan, M. Z. Uddin, A. Mohamed, and A. Almogren, "A robust human activity recognition system using smartphone sensors and deep learning," Future Generation Computer Systems., vol. 81, pp. 307-313, 2018.

[19] D. Acharjee, S. P. Maity, and A. Mukherjee, "Hidden Markov model a tool for recognition of human contexts using sensors of smart mobile phone," Microsystem Technologies., no. August, pp. 1-12, 2016.

[20] D. Acharjee, A. Mukherjee, J. K. Mandal, and N. Mukherjee, "Activity recognition system using inbuilt sensors of smart mobile phone and minimizing feature vectors," Microsystem Technologies., vol. 22, no. 11, pp. 2715-2722, Nov. 2016.

[21] J. a Gallian, J. G. Rainbolt, and B. Cole, Abstract Algebra with GAP for Contemporary Abstract Algebra, 2010.

[22] M. Eie and S.-T. Chang, "Cyclic Groups," A Course Abstr. Algebr., pp. 53-64, 2012.

[23] A. Cyclic and G. Structures, "As Cyclic Group Structures," no. $\mathrm{M}, 2014$.

[24] S. Spitzer, "Using Star Polygons to Understand Cyclic Group Structure," pp. 479-480, 2012.

[25] M. M. El-farrah, "Expectation Numbers of Cyclic Groups," 2015.

[26] H. Aktaş and Ş. Özlü, "Cyclic soft groups and their applications on groups," Scientific World Journal., vol. 2014, 2014.

[27] G. Oman and V. Slattum, "A Characterization of the Cyclic Groups by Subgroup Indices," Coll. Math. J., vol. 47, no. 1, pp. 29-33, 2016.

\section{Authors' Profiles}

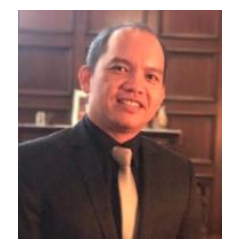

Pattara Aiyarak, is a computer and technology enthusiast. He received degrees in various fields such as bachelor's degrees in economics, Banking and Finance, Physics and, of course, Ph.D. in Physics (Telecommunications). His background reflects his broad research interest in several areas. He recently works as an assistant professor in the department of Computer Science, Faculty of Science, Prince of Songkla University. He has supervised several postgraduate students in Forensic Science, MIS, Physics and Computer Science.

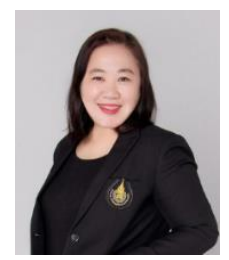

Wiphada Wettayaprasit, is an assistant professor at Department of Computer Science, Faculty of Science, Prince of Songkla University, Thailand. She got BSc., MSc., PhD. in Computer Science from Prince of Songkla University, University of Missouri-Columbia, USA, and Chulalongkorn University, respectively. Her research interests are Artificial Intelligence, Neural Networks, and Machine Learning.

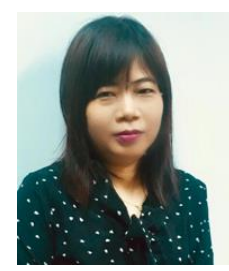

Win Win Myo, is a $\mathrm{PhD}$. candidate at Department of Computer Science, Faculty of Science, Prince of Songkla University, Thailand and currently an associate professor at Faculty of Computing, University of Information Technology, Yangon, Myanmar. She got her BSc. (Mathematics), MSc. (Mathematics), MSc. (Computer Science) from Yangon University, Myanmar. Her research interests are Human Activity Recognition and Machine Learning.

How to cite this paper: Win Win Myo, Wiphada Wettayaprasit, Pattara Aiyarak, "A Cyclic Attribution Technique Feature Selection Method for Human Activity Recognition", International Journal of Intelligent Systems and Applications(IJISA), Vol.11, No.10, pp.25-32, 2019. DOI: 10.5815/ijisa.2019.10.03 